\title{
CARACTERIZAÇÃo QUÍMICA DOS GÉIS PRODUZIDOS PELAS BACTÉRIAS DIAZOTRÓFICAS Rhizobium tropici E Mesorhizobium sp.
}

Nilson Kobori Monteiro

Departamento de Engenharia e Tecnologia de Alimentos, Instituto de Biociências, Letras e Ciências Exatas, Universidade Estadual Paulista, 15054-000 São José do Rio Preto - SP, Brasil

Gabriel Aranda-Selverio, Diego Tadeu Degli Exposti e Maria de Lourdes Corradi da Silva*

Departamento de Física, Química e Biologia, Faculdade de Ciências e Tecnologia, Universidade Estadual Paulista, 19060-900

Presidente Prudente - SP, Brasil

Eliana Gertrudes Macedo Lemos e João Carlos Campanharo

Departamento de Tecnologia, Faculdade de Ciências Agrárias e Veterinária, Universidade Estadual Paulista, 14884-900 Jaboticabal - SP, Brasil

Joana Léa Meira Silveira

Departamento de Bioquímica e Biologia Molecular, Universidade Federal do Paraná, 81531-990 Curitiba - PR, Brasil

Recebido em 6/5/11; aceito em 1/11/11; publicado na web em 4/1/12

CHEMICAL CHARACTERIZATION OF THE GELS PRODUCED BY THE DIAZOTROPHIC BACTERIA Rhizobium tropici AND Mesorhizobium sp. The exopolysaccharides with characteristics of gel produced by Rhizobium tropici $\left(\mathrm{EPS}_{\mathrm{RT}}\right.$ ) and Mesorhizobium $\mathrm{sp}$ $\left(\mathrm{EPS}_{\mathrm{MR}}\right)$ are acidic heteropolysaccharide composed mainly of glucose and galactose in a molar ratio of 4:1 and 5:1 respectively, with traces of mannose $(\sim 1 \%)$. Chemical analysis showed the presence of uronic acid, pyruvate and acetyl-substituents in the structures of both polymers. Experiments of gel permeation chromatography and polyacrylamide gel electrophoresis showed that EPS $\mathrm{RT}_{\mathrm{RT}}$ and $\mathrm{EPS}_{\mathrm{MR}}$ are homogeneous molecules with low grade of polydispersity. The EPS were characterized using spectroscopic techniques of FT-IR, ${ }^{1} \mathrm{H}$ and ${ }^{13} \mathrm{C}-\mathrm{NMR}$.

Keywords: acid exopolysaccharide; Rhizobium tropici; Mesorhizobium sp.

\section{INTRODUÇÃO}

Juntamente com os ácidos nucleicos e as proteínas, os polissacarídeos determinam a funcionalidade e especificidade de uma espécie, encontram-se amplamente disseminados na natureza e possuem uma estrutura altamente organizada. ${ }^{1}$ A estrutura primária de um polissacarídeo é definida pela composição, sequência e tipo de monossacarídeos presentes. ${ }^{2}$ Considerando que algumas dessas moléculas se comportam como géis estáveis quando em solução aquosa, a demanda por polímeros com essa característica tem aumentado consideravelmente nos últimos anos. ${ }^{3}$

Diversos micro-organismos são capazes de sintetizar e secretar polissacarídeos como compostos solúveis ou insolúveis em meio aquoso. ${ }^{3}$ Dentre eles estão as bactérias diazotróficas que são responsáveis pela fixação biológica de nitrogênio, convertendo o nitrogênio molecular atmosférico $\left(\mathrm{N}_{2}\right)$ em íon amônio $\left(\mathrm{NH}_{4}^{+}\right)$. As bactérias diazotróficas da família Rhizobeaceae, ${ }^{4}$ constituída pelos gêneros Rhizobium, Sinorhizobium, Mesorhizobium, Bradyrhizobium e Azorhizobium, ${ }^{5}$ são investigadas por produzirem quantidades consideráveis de exopolissacarídeos que utilizam para sua fixação nas raízes de plantas leguminosas, em um processo que facilita a obtenção de nutrientes e fornece proteção contra o ambiente e as defesas do hospedeiro. ${ }^{6}$

Dentre os polissacarídeos secretados por essas bactérias encontram-se as succinoglucanas, poliglucurananas e glucanas cíclicas. ${ }^{7}$ Os polissacarídeos ácidos são mais investigados por apresentarem propriedades reológicas e farmacológicas diferenciadas ${ }^{8}$ e, consequentemente, despertarem o interesse das indústrias alimentícia e farmacêutica. Há relatos na literatura que esses polímeros são cons-

*e-mail: corradi@fct.unesp.br tituídos por D-glucose, D-galactose e ácido D-glucurônico, podendo apresentar piruvato, geralmente ligado aos resíduos terminais das ramificações, e grupos $O$-acetil. ${ }^{9}$

Aranda-Selverio e colaboradore ${ }^{10}$ realizaram alguns estudos reológicos com soluções de exopolissacarídeos produzidos pelas bactérias Rhizobium tropici (diferentes linhagens) e Mesorhizobium sp e verificaram que essas soluções apresentam comportamento de fluxo e viscosidade distintas, sendo, provavelmente, a composição química desses compostos responsável por essas diferenças. Para confirmar essa suposição dois dos quatro EPS estudados por Aranda-Selverio e colaboradores ${ }^{10}$ foram selecionados para estudo químico neste trabalho. As razões para isso foram as características de material homogêneo apresentadas pelos EPS produzidos por uma linhagem de Rhizobium tropici e do Mesorhizobium sp denominados, respectivamente, de $\mathrm{EPS}_{\mathrm{RT}}$ e $\mathrm{EPS}_{\mathrm{MR}}$.

\section{PARTE EXPERIMENTAL}

\section{Materiais}

Os EPS produzidos pelas bactérias Rhizobium tropici $\left(\mathrm{EPS}_{\mathrm{RT}}\right)$ e Mesorhizobium $\left(\mathrm{EPS}_{\mathrm{MR}}\right.$ ) foram fornecidos pela Profa. Dra. E. G. de M. Lemos - FCAV/UNESP Jaboticabal, São Paulo, Brasil. As informações sobre as condições de cultivo estão descritas na patente registrada como PI0304053-4.

\section{Métodos analíticos}

Açúcares totais e redutores foram determinados pelos métodos do fenol-ácido sulfúrico ${ }^{11}$ e de Somogy e Nelson, ${ }^{12}$ respectivamente, e a glucose foi utilizada como padrão em ambos os ensaios. As proteínas 
foram determinadas pelo método de Bradford ${ }^{13}$ e soro albumina bovina foi utilizada como padrão. Os ácidos urônicos foram quantificados pelo método de Blumenkrants modificado ${ }^{14} \mathrm{e}$ o ácido glucurônico foi utilizado como padrão. A quantificação de grupos acetil foi realizada pelo método de Hestrin ${ }^{15}$ e o cloreto de acetilcolina foi utilizado como padrão. O grau de substituição por grupos piruvato foi determinado pelo método de Sloneker e Orentas ${ }^{16}$ e o ácido pirúvico foi utilizado como padrão.

\section{Preparo dos polissacarídeos}

Os $\mathrm{EPS}_{\mathrm{RT}}$ e $\mathrm{EPS}_{\mathrm{MR}}$ foram, separadamente, dialisados em membranas de celulose de $12 \times 10^{3} \mathrm{Da}$ (massa molecular de corte - MWCO), contra água destilada corrente, por $72 \mathrm{~h}$, para a remoção de resquícios do meio de cultura e, então, liofilizados e estocados sob refrigeração.

\section{Análise da homogeneidade e da massa molecular aparente por HPSEC}

A homogeneidade dos polissacarídeos foi determinada por cromatografia de gel permeação em cromatógrafo líquido de alta pressão, marca Shimadzu, com detector de índice de refração diferencial modelo RID 10A, usando quatro colunas de gel permeação da marca Waters, com limites de exclusão de $7 \times 10^{6}, 4 \times 10^{5}, 8 \times 10^{4}$ e $5 \times 10^{3} \mathrm{Da}$, dispostas em série. A determinação das massas moleculares aparentes foi realizada utilizando-se uma curva de calibração com padrões de dextrana com massas moleculares variando de $9,4 \times 10^{3}$ a 2, $1 \times 10^{6} \mathrm{Da}$.

\section{Eletroforese em gel de poliacrilamida (PAGE)}

As análises eletroforéticas foram realizadas em sistema Hoefer ${ }^{\circledR}$ mini VE (Pharmacia Biotech), utilizando gel de poliacrilamida com espessura de $75 \mathrm{~mm}$, constituído de uma região de gel concentrador (4\%) de 14 mm com tampão tris- $\mathrm{HCl}$ 0,5M pH 6,8, e uma camada de gel separador (6\%) de $83 \mathrm{~mm}$ com tampão tris- $\mathrm{HCl} 1,5 \mathrm{M} \mathrm{pH} 8,8$. Foram aplicados $2 \mu \mathrm{L}$ da solução $(5 \mathrm{mg} / \mathrm{mL})$ de cada um dos EPS. A corrida eletroforética foi efetuada em tampão fosfato $0,02 \mathrm{M} \mathrm{pH} 7,6$, tensão de $110 \mathrm{~V}$ por $1 \mathrm{~h} 45 \mathrm{~min}$. O gel foi corado com solução $0,1 \%$ (m/v) de azul de toluidina em ácido acético $5 \%(\mathrm{v} / \mathrm{v})$ por $5 \mathrm{~min}$ e, então, foi lavado com água destilada até a remoção do excesso de azul de toluidina.

\section{Análise de monossacarídeos por HPAEC}

Para análise dos monossacarídeos, alíquotas de $50 \mu \mathrm{g}$ de açúcares totais, de cada um dos EPS, foram hidrolisadas com TFA 2M a $121{ }^{\circ} \mathrm{C}$, por $2 \mathrm{~h}$. $\mathrm{O}$ ácido foi removido em evaporador rotativo, à baixa pressão, e solubilizado em água deionizada. Frações contendo cerca de $1 \mu \mathrm{g}$ de açúcares totais em $25 \mu \mathrm{L}$ de água deionizada foram injetadas em HPAEC (Dionex DX500) equipado com um detector de amperometria pulsada (PAD). Os açúcares neutros foram separados isocraticamente, usando uma coluna analítica CarboPac PA1 equipada com guarda coluna PA1, ao fluxo de $1 \mathrm{~mL} / \mathrm{min}$. As condições de eluição foram produzidas utilizando $\mathrm{H}_{2} \mathrm{O}$ deionizada (eluente 1) e $7 \% \mathrm{NaOH} 200 \mathrm{mM}$ (eluente 2), resultando em um fase móvel de $\mathrm{NaOH} 14$ mM, com corridas de 25 min de duração.

\section{Análise dos espectros de FT-IR}

Os espectros de FT-IR (3800 a $500 \mathrm{~cm}^{-1}$ ) dos EPS $_{\mathrm{RT}}$ e $\mathrm{EPS}_{\mathrm{MR}}$ foram obtidos em espectrômetro BRUKER modelo Vector $22 \mathrm{com}$ resolução de $4 \mathrm{~cm}^{-1}$. A análise foi realizada nas pastilhas formada por $1 \mathrm{mg}$ de cada EPS macerado com $250 \mathrm{mg}$ de $\mathrm{KBr}$ e com a aplicação de uma pressão de $5,5 \mathrm{t}$ por $3 \mathrm{~min}$.

\section{Análise dos espectros de $\mathrm{RMN}$ de ${ }^{1} \mathrm{H}$ e ${ }^{13} \mathrm{C}$}

Os espectros de RMN de prótons $\mathrm{e}^{13} \mathrm{C}$ foram obtidos em espectrômetro Bruker, modelo Avance-DRX-400. Os EPS foram solubilizados em óxido de deutério, armazenados em tubos de 100 x $10 \mathrm{~mm}$ d.i. e mantidos a $70{ }^{\circ} \mathrm{C}$ durante a análise. Os espectros foram obtidos em $400 \mathrm{MHz}$ em relação ao núcleo de ${ }^{1} \mathrm{H}(100 \mathrm{MHz}$ em relação ao núcleo de ${ }^{13} \mathrm{C}$ ). Os deslocamentos químicos foram expressos em $\delta$ (ppm) relativos aos sinais de ${ }^{13} \mathrm{C} \mathrm{e}{ }^{1} \mathrm{H}$ da acetona em $\delta 30,20$ e 2,22, respectivamente.

\section{RESULTADOS E DISCUSSÃO}

\section{Quantificação dos exopolissacarídeos}

Durante a síntese dos EPS pelos micro-organismos, há parâmetros que podem influenciar no rendimento e na composição monossacarídica dessas moléculas, ${ }^{17}$ acarretando diferenças nas propriedades físico-químicas de suas soluções. Os exopolissacarídeos $\left(\mathrm{EPS}_{\mathrm{RT}}\right.$ do Rhizobium tropici e EPS $_{\mathrm{MR}}$ do Mesorhizobium $\mathrm{sp}$ ) foram produzidos sob as mesmas condições de cultivo e a determinação e quantificação de seus constituintes principais foram efetuadas. Os teores de açúcares totais para $\operatorname{EPS}_{\mathrm{RT}}(85 \%)$ e $\operatorname{EPS}_{\mathrm{MR}}(80 \%)$ e o baixo percentual de açúcar redutor $(0,1 \%)$ indicam que, praticamente, todo o carboidrato presente nas amostras se encontra na forma de polissacarídeo. A detecção de $4 \%$ de proteínas, em ambos, pode estar relacionada à presença de uma exoenzima produzida pelos micro-organismos que, entretanto, não foi investigada neste trabalho. As análises para ácido urônico, acetil e piruvato confirmam que são polímeros ácidos, sendo a diferença mais significativa em relação aos teores de piruvato e ácido urônico, 6 e 2\% para o EPS $_{\mathrm{RT}}$ e 1 e $13 \%$ para o $\mathrm{EPS}_{\mathrm{MR}}$. Foram 3 e $2 \%$ os valores encontrados para os substituintes acetil nos $\mathrm{EPS}_{\mathrm{RT}}$ e EPS $\mathrm{MR}_{\mathrm{R}}$, respectivamente. Provavelmente essas diferenças na composição sejam, em parte, responsáveis pelos comportamentos reológicos distintos ${ }^{18}$ de suas soluções, fornecendo géis fortes para EPS $_{\mathrm{RT}}$ e fracos para $\mathrm{EPS}_{\mathrm{MR}} \cdot{ }^{10}$

\section{Determinação da homogeneidade e da massa molecular aparente}

Para verificar a homogeneidade das amostras foram realizados ensaios cromatográficos e eletroforéticos (Figura 1S, material suplementar). Os resultados da cromatografia de filtração em gel indicaram, através da detecção de um único pico, que ambos os EPS se apresentavam como moléculas homogêneas, sendo o exopolissacarídeo produzido pelo Rhizobium tropici menos polidisperso. As massas moleculares aparentes dos $\mathrm{EPS}_{\mathrm{RT}}$ e $\mathrm{EPS}_{\mathrm{MR}}$ foram estimadas em $32,2 \times 10^{6}$ e $19,1 \times 10^{6} \mathrm{Da}$, respectivamente, por regressão linear dos tempos de retenção e dos logaritmos das massas moleculares aparentes de padrões de dextrana.

Considerando-se que ambos os EPS contêm grupos ionizáveis, ${ }^{19} \mathrm{a}$ eletroforese em gel de poliacrilamida foi escolhida como um segundo método para verificar a homogeneidade dos polímeros, cujo perfil confirmou os resultados encontrados na cromatografia de filtração em gel.

\section{Determinação da composição monossacarídica}

A hidrólise ácida e análise por HPAEC/PAD dos EPS $_{\mathrm{RT}}$ e $\mathrm{EPS}_{\mathrm{MR}}$ apresentaram glucose e galactose nas relações molares de 4:1 e 5:1, respectivamente. Também foram detectados traços de manose em ambos (1\% para $\mathrm{EPS}_{\mathrm{RT}}$ e $3 \%$ para $\mathrm{EPS}_{\mathrm{MR})}$. Estes dados são semelhantes aos encontrados por Castellane e $\operatorname{Lemos}^{20}$ que estudaram um EPS proveniente do cultivo de Rhizobium tropici SEMIA 4077, 
com glucose e galactose como componentes majoritários e traços de manose e ramnose.

\section{Análises de infravermelho com transformada de Fourier dos EPS $_{\mathrm{RT}}$ e EPS $_{\mathrm{MR}}$}

Nos espectros de FT-IR mostrados na Figura 1a, são observados sinais em $1750 \mathrm{~cm}^{-1}$ referentes às carboxilas dos ácidos urônicos e pirúvico. ${ }^{21}$ Sinais em 895 e $900 \mathrm{~cm}^{-1}$ para $\mathrm{EPS}_{\mathrm{RT}}$ e $\mathrm{EPS}_{\mathrm{MR}}$, respectivamente, presentes na região de impressão digital do espectro (Figura 1b) indicam a presença de ligações glicosídicas do tipo $\beta$ em ambos. ${ }^{22} \mathrm{O}$ espectro de FT-IR do polissacarídeo produzido pela bactéria Mesorhizobium sp apresenta uma leve deformidade próximo a $850 \mathrm{~cm}^{-1}$, provavelmente, referente às vibrações associadas a ligações do tipo $\alpha .^{23}$

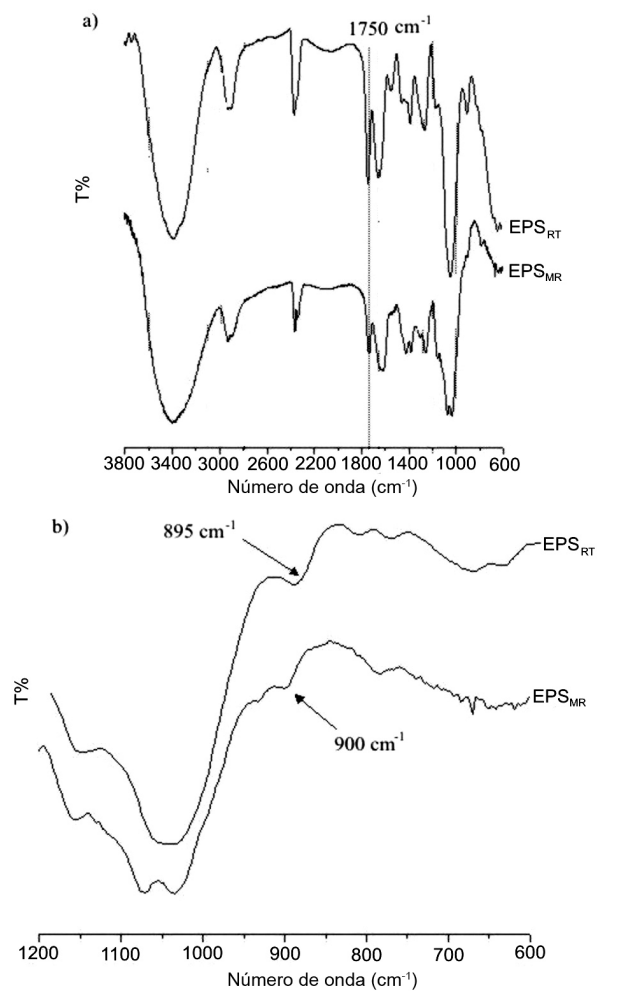

Figura 1. Espectros de FT-IR dos $E P S_{R T}$ e $E P S_{M R}$

\section{Análises de ressonância magnética nuclear de ${ }^{1} \mathrm{H}$ e ${ }^{13} \mathrm{C}$ do EPS $_{\text {RT }}$}

No espectro de RMN de ${ }^{1} \mathrm{H}$ do $\operatorname{EPS}_{\mathrm{RT}}$ (Figura 2S-a, material suplementar) sinais intensos em 1,47 e 2,06 ppm foram atribuídos aos hidrogênios metílicos presentes nos grupos piruvato e acetil $O$ -ligado. ${ }^{9}$ A ausência de sinais entre 2,50 a 3,00 ppm, relacionados aos prótons metilênicos, ${ }^{24}$ sugere que não há succinil na molécula. Os sinais de prótons que constituem o anel heterocíclico, com exceção dos anoméricos, estão entre 3,40 e 4,50 ppm e aquele mais intenso em 3,49 ppm pode ser resultante da sobreposição dos sinais de $\mathrm{H}-5$ e dos hidrogênios do grupo metil 4-O-ligado que pode estar presente no ácido glucurônico. ${ }^{25}$ Os deslocamentos químicos entre 4,52 a 4,81 ppm, atribuídos aos prótons anoméricos, confirmam que o $\mathrm{EPS}_{\mathrm{RT}}$ tem suas unidades monossacarídicas predominantemente em configuração $\beta$.

$\mathrm{O}$ espectro de $\mathrm{RMN}$ de ${ }^{13} \mathrm{C}$ do $\mathrm{EPS}_{\mathrm{RT}}$ (Figura 2S-b, material suplementar) apresenta sinais em 20,3 e 24,7 ppm atribuídos, respec- tivamente, aos grupos metílicos do acetil e do piruvato. ${ }^{8} \mathrm{O}$ sinal em 57,4 ppm está associado ao grupo metil- $O$-ligado ao ácido urônico, ${ }^{25}$ confirmando a atribuição efetuada no espectro de prótons. Quatro sinais na região de C-6 livre podem indicar que o polímero possui quatro unidades com ambientes químicos distintos. Segundo Rinaudo e colaboradores ${ }^{26}$ há deslocamento dos sinais de C-6 livre para campo mais baixo quando grupamentos acetil ou piruvato se ligam à molécula $(60,5 \rightarrow 63,7 \mathrm{ppm}, 61,1 \rightarrow 64,3 \mathrm{ppm})$. Os sinais em $68,5 \mathrm{e}$ 68,9 ppm são atribuídos aos C-6- $O$-substituídos envolvidos na ligação glicosídica. ${ }^{27}$ Sinais em 79,0/79,2 e em 84,8/85,1 ppm são atribuídos aos C-4- $O$ - e C-3- $O$-substituídos, respectivamente. ${ }^{28}$ Exopolissacarídeos produzidos por bactérias da família Rhizobeaceae possuem ligações do tipo $1 \rightarrow 3$ e $1 \rightarrow 4$ entre os resíduos monossacarídicos da cadeia principal ${ }^{6,29}$ e ligações $1 \rightarrow 6$, geralmente associadas aos pontos de ramificação. ${ }^{9} \mathrm{Na}$ região de carbonos anoméricos, os sinais entre 103,4 a 102,6 ppm estão relacionados aos C-1 das unidades $\beta$-D-glucopiranosídicas, $\beta$-D-galactopiranosídicas (substituídas ou não com piruvato) e ácido urônico. Nessa mesma região do espectro se encontra o sinal de deslocamento químico do carbono quaternário do piruvato (C-2) resultante da ligação do composto à estrutura polissacarídica. ${ }^{26}$ Os sinais em 173,7 e 174,9 ppm foram atribuídos às carboxilas presentes nos ácidos urônico e pirúvico, respectivamente. ${ }^{30}$

Nas condições de hidrólise realizadas neste trabalho não foi detectado deoxiaçucar, entretanto, Castellane e $\operatorname{Lemos}^{20}$ encontraram traços de ramnose $(\sim 0,1 \% \mathrm{~m} / \mathrm{m})$ em polissacarídeos produzidos por Rhizobium tropici SEMIA 4077 e SEMIA 4080. Portanto, o sinal em 16,9 ppm, normalmente associado ao carbono de grupo metila de deoxiaçúcares, e um pequeno sinal em 5,40 ppm encontrado no espectro de $\mathrm{RMN}-{ }^{1} \mathrm{H}$, atribuído aos anômeros $\alpha$, podem estar relacionados com a presença de $\alpha$-L- ramnose.

A partir do exposto propõe-se que o $\mathrm{EPS}_{\mathrm{RT}}$ seja formado por blocos pentassacarídicos repetitivos que se constituem por três unidades $\beta$-D-glucopiranosídicas alternadas por ligações do tipo $(1 \rightarrow 3)$ e $(1 \rightarrow 4)$ e ramificações em $(1 \rightarrow 6)$ de $\beta$-D-glucopiranose e galactopiranose, estando algumas desta última unidade 4,6-O-substituídas pelo grupo piruvato. Também é possível propor que algumas unidades pentassacarídicas tenham um resíduo de glucose substituído pelo ácido glucurônico. A posição do grupo acetil não foi determinada.

\section{Análises de ressonância magnética nuclear de ${ }^{1} \mathrm{H}$ e ${ }^{13} \mathrm{C}$ do $\mathrm{EPS}_{\mathrm{MR}}$}

No espectro de RMN de ${ }^{1} \mathrm{H}$ do $\mathrm{EPS}_{\mathrm{MR}}$ (Figura 3S-a, material suplementar) os sinais em 1,49 e 1,93 ppm correspondem aos hidrogênios metílicos presentes nos grupos piruvato e acetil $O$-ligados. ${ }^{9} \mathrm{~A}$ ausência de sinais entre 2,5 a 3,0 ppm mostra que o EPS não apresenta grupos succinil em sua estrutura. Na região de prótons anoméricos os sinais em 5,31 e 5,05 ppm foram atribuídos às unidades ácido $\alpha$-D-glucurônicos e -D-galactofuranosídicas, ${ }^{31}$ respectivamente. O deslocamento em $5,15 \mathrm{ppm}$ pode ser atribuído às unidades de galf$O$-4-6-Pyr que ocorrem em pequena quantidade.

No espectro de $\mathrm{RMN}{ }^{13} \mathrm{C}$ (Figura 3S-b, material suplementar) do polissacarídeo produzido pela bactéria Mesorhizobium o sinal em 20,3 ppm foi atribuído ao grupo metílico presente no acetil, 8,30 não foi detectado sinal na região de $24,5 \mathrm{ppm}$, referente ao carbono do ácido carboxílico do piruvato, possivelmente oculto pelo ruído da linha de base. Os deslocamentos químicos em 60,1 e 60,9ppm correspondem aos C-6 livres das unidades -D-galactofuranosídicas ${ }^{31}$ e -D-glucopiranosídicas, respectivamente. Os sinais em 69,4 e 69,9 ppm correspondem às unidades C-6- $O$-substituídas, indicando a presença de ligações $(1 \rightarrow 6)$. O sinal em $77,9 \mathrm{ppm}$ corresponde ao C-5- $O$-substituído de unidades galactofuranosídicas, ${ }^{31,32}$ semelhante em intensidade ao 107,6 ppm, atribuído ao carbono anomérico desses monômeros. Unidades -D-galactofuranosídicas podem ser encontra- 
das em polissacarídeos bacterianos. ${ }^{32}$ Os deslocamentos químicos em 82,4/81,8 e 79,5/78,5 ppm foram atribuídos aos C-3- $O$ e C-4- $O$ substituídos, ${ }^{33}$ respectivamente. O sinal em 99,8 ppm corresponde ao C-1 do ácido $\alpha$-D-glucurônicos ${ }^{34}$ e em 102,7/103,2 ppm aos C-1 de unidades -D-glucopiranosídicas. ${ }^{22,35}$ Os deslocamentos químicos em campo baixo, 174,8 e 173,6/172,7 ppm foram atribuídos às carboxilas do piruvato e dos ácidos urônicos ${ }^{30}$ respectivamente. Esses dois últimos sinais podem indicar que há duas unidades distintas de ácido urônico na composição da cadeia polissacarídica.

Pela complexidade dos resultados e falta de informação na literatura sobre exopolissacarídeos produzidos por Mesorhizobium, não foi possível propor a estrutura deste polímero sem o auxílio da ressonância magnética nuclear bidimensional. Entretanto, com os dados obtidos até o momento é possível afirmar que as estruturas dos exopolissacarídeos produzidos pelos Rhizobium tropici e Mesorhizobium são bastante diferentes, podendo ser um dos motivos pelos quais suas soluções apresentam propriedades físico-químicas distintas.

\section{CONCLUSÕES}

Os EPS foram caracterizados como compostos ácidos, homogêneos e pouco polidispersos com massas moleculares aparentes superiores a $2 \times 10^{6} \mathrm{Da}$. O EPS $\mathrm{RT}_{\mathrm{RT}}$ ć constituído por glucose e galactose nas relações molares de 4:1 com $2 \%$ de ácido urônico e substituição

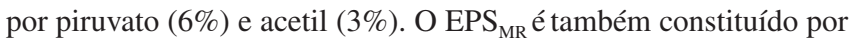
glucose e galactose numa relação molar de 5:1, com 13\% de ácido urônico e 1 e $2 \%$ de piruvato e acetil, respectivamente. Análises de $\mathrm{RMN}$ de ${ }^{1} \mathrm{H} \mathrm{e}{ }^{13} \mathrm{C}$ mostraram que o $\mathrm{EPS}_{\mathrm{RT}}$ tem estrutura química semelhante a alguns exopolissacarídeos de bactérias do gênero Rhizobium, embora sem a presença de succinil. O exopolissacarídeo produzido pelo Mesorhizobium tem em sua composição monossacarídica unidades de galactose na forma furanosídica e ácido urônico em configuração $\alpha$.

\section{MATERIAL SUPLEMENTAR}

Figuras referentes aos testes de homogeneidade (cromatografia de filtração em gel e eletroforese) e aos espectros de $\mathrm{RMN}-{ }^{13} \mathrm{C}$ e $\mathrm{RMN}-{ }^{1} \mathrm{H}$ são encontradas como material suplementar, em http://quimicanova. sbq.org.br, em arquivo PDF com acesso livre.

\section{AGRADECIMENTO}

À CAPES pela bolsa de mestrado ao aluno N. K. Monteiro.

\section{REFERÊNCIAS}

1. Kajiwara, K.; Miyamoto, T. Em Polysaccharides - structural diversity and functional versatility; Severian, D., ed.; Marcel Dekker: Nova Iorque, 2005, cap. 1.

2. Pazur, J. H. Em Carbohydrate Analysis: A Practical Approach; Chaplin, M. F.; Kennedy, J. F., eds.; Oxford University Press: Oxford, 1994, cap. 4.

3. Kumar, A. S.; Mody, K.; Jha, B.; J. Basic Microbiol. 2007, 47, 103.

4. Prévost, D.; Antoun, H. Em Soil Sampling and Methods of Analysis; Carter, M. R.; Gregorich, E. G., eds.; CRC Press: Londres, 2007, cap. 4.

5. Werner, D. Em Biochemistry and Organic Substances at the Soil-Plant Interface; Pinton, R.; Varanini, Z.; Nannipieri, P., eds.; CRC Press: Londres, 1997, cap. 8.
6. York, G. M.; Walker, G. C.; J. Bacteriol. 1998, 180, 4184.

7. Guentas, L.; Pheulpin, P.; Heyraud, A.; Gey, C.; Courtois, B.; Courtois, J.; Int. J. Biol. Macromol. 2000, 27, 269; Courtois, J.; Seguin, J.-P.; Roblot, C.; Heyraud, A.; Gey, C.; Dantas, L.; Barbotin, J.-N.; Courtois, B.; Carbohydr. Polym. 1994, 25, 7; Heyraud, A.; Courtois, S.; Dantas, L.; Colin-Morel, P.; Courtois, B.; Carbohydr. Res. 1993, 240, 71; Breedveld, M. W.; Miller, K. J.; Microbiol. Rev. 1994, 58, 145.

8. Zhao, L.; Chen, Y.; Ren, S.; Han, Y.; Cheng, H.; Carbohydr. Res. 2010, 345, 637.

9. Mcneil, M.; Darvill, J.; Darvill, A. G.; Albersheim, P.; Veen, R. V.; Hooykaas, P.; Schilperoort, R.; Dell, A.; Carbohydr. Res. 1986, 146, 307; Ridout, M. J.; Brownsey, G. J.; York, G. M.; Walker, G. C.; Morris, V. J.; Int. J. Biol. Macromol. 1997, 20, 1.

10. Aranda-Selverio, G.; Penna, A. L. B.; Campos-Sás, L. F.; Santos Jr., O.; Vasconcelos, A. F. D.; da Silva, M. L. C.; Lemos, G. M.; Campanaro, J. C.; Silveira, J. L. M.; Quim. Nova 2010, 33, 895.

11. Dubois, N.; Gilles, K. A.; Hamilton, J. K.; Rebers, P. A.; Smith, F.; Anal. Chem. 1956, 28, 350

12. Somogyi, M.; J. Biol. Chem. 1945, 160, 61; Nelson, N. A.; J. Biochem. 1944, 153, 376

13. Bradford, M. M.; Anal. Biochem. 1976, 72, 248.

14. Filisetti-Cozzi, T. M. C.; Carpita, N. C.; Anal. Biochem. 1991, 197, 157.

15. Hestrin, S.; J. Biol. Chem. 1949, 180, 879.

16. Sloneker, J. H.; Orentas, A.; Can. J. Chem. 1962, 40, 2066.

17. Souza, D. M.; Garcia-Cruz, C. H.; Semina ciênc. agrar. 2004, 25, 331.

18. Rinaudo, M.; Biomacromolecules 2004, 5, 1155.

19. Zierer, M. S.; Mourão, P. A. S.; Carbohydr. Res. 2000, 328, 209.

20. Castellane, T. C. L.; Lemos, E. G. M.; Pesq. Agropec. Bras. 2007, 43, 1503.

21. Vuyst, L. D.; Degeest, B.; FEMS Microbiol. Rev. 1999, 23, 153.

22. Vasconcelos, A. F. D.; Monteiro, N. K.; Dekker, R. F. H.; Barbosa, A. M.; Carbonero, E. R.; Silveira, J. L. M.; Sassaki, G. L.; Silva, R.; Corradi da Silva, M. L.; Carbohydr. Res. 2008, 343, 2481.

23. Zhao, G.; Kan, J.; Li, Z.; Chen, Z.; Int. Immunopharmacol. 2005, 5, 1436 .

24. Simsek, S.; Mert, B.; Campanella, O. H.; Reuhs, B.; Carbohydr. Polym. 2009, 76, 320.

25. Dudman, F. W.; Carbohydr. Res. 1978, 66, 9.

26. Rinaudo, M.; Vincendon, M.; Carbohydr. Polym. 1982, 2, 135.

27. Cui, S.W. Em Food Carbohydrates, Chemistry, Physical Properties, and Applications; Cui, S. W., ed.; CRC Press: Londres, 2005, cap. 3.

28. Yang, L.; Zhang, L.-M.; Carbohydr. Polym. 2009, 76, 349.

29. Carlson, R. W.; Reuhs, B. L.; Forsberg, L. S.; Kannenberg, E. L. Em Genetics of Bacterial Polysaccharides; Goldberg, J. B., ed.; CRC Press: Londres, 1999, cap. 3.

30. Urai, M.; Yoshizaki, H.; Anzai, H.; Ogihara, J.; Iwabuchi, N.; Harayama, S.; Sunairi, M.; Nakajima, M.; Carbohydr. Res. 2007, 342, 933.

31. Ahrazem, O.; Leal, J. A.; Prieto, A.; Jiménez-Barbero, J.; Bernabé, M.; Carbohydr. Res. 2001, 336, 325; Prieto, A.; Leal, J. A.; Gómez-Miranda, B.; Ahrazem, O.; Jiménez-Barbero, J.; Bernabé, M.; Carbohydr. Res. 2001, 333, 173.

32. Lemercinier, X.; Jones, C.; Carbohydr. Res. 2006, 341, 68.

33. Chouly, C.; Colquhoun, I. J.; Jodelet, A.; York, G.; Walker, G. C.; Int. J. Biol. Macromol. 1995, 17, 357.

34. Aono, R.; Biochem. J. 1990, 270, 363.

35. Corradi Da Silva, M. L.; Izeli, N. L.; Martinez, P. F.; Silva, I. R.; Constantino, C. J. L.; Cardoso, M. S.; Barbosa, A. M. H.; Dekker, R. F.; Silva, G. V. J.; Carbohydr. Polym. 2005, 61, 10. 